\title{
M-ESTIMATION AS A TOOL SUPPORTING A VESSEL TRAFFIC CONTROLLER IN THE VTS SYSTEM
}

\author{
Sławomir Świerczyński ${ }^{1}$ \\ Krzysztof Czaplewski ${ }^{2}$ \\ 1) Polish Naval Academy, Poland \\ 2) Gdynia Maritime University, Poland
}

\begin{abstract}
In order to improve maritime safety and the efficiency of vessel traffic, systems supervising vessel traffic, i.e. VTS (Vessel Traffic Service), started to be created. These systems are aimed to control vessel traffic in waters where traffic congestion, a large concentration of vessels or the presence of navigational hazards creates a risk of collision or stranding. VTS systems constitute maritime safety centres and they must be equipped with appropriate devices in order to be fully functional. Among devices that provide information about vessels are coastal radar stations which are located around a monitored sea area. This kind of spatial arrangement of these stations can be used to simultaneously obtain information about every vessel, but such observations may be fraught with serious errors. Therefore, the estimation methods that are employed and developed in geodesy can be used to improve the accuracy with which a vessel's position is determined. The Interactive Navigational Structure, i.e. IANS, is an example of how these methods can be applied in navigation; this term has already been introduced into the literature (Czaplewski, 2004). The text below presents the theoretical assumptions underlying the use of IANS as a tool supporting a vessel traffic controller using the VTS system in his/her work. This presentation is supported by a numerical test that was performed in the waters of the Bay of Gdańsk which are covered by the VTS system.
\end{abstract}

Keywords: navigation, radar navigation; Vessel Traffic Services; M-estimation; Interactive Navigational Structures

\section{INTRODUCTION}

Since the volume of vessel traffic is constantly growing, increasing emphasis should be placed on maritime safety. Marine traffic safety systems function in many sea areas; these systems are mainly aimed to improve the safety of ship transport, protect the marine environment and respond to emergencies. Sea areas are monitored by a network of coastal radar stations which constitute one of the main sources of information about vessels. Radars are to detect not only large vessels entering zones that are monitored by the VTS system, but also small ones, such as sports and fishing vessels. If the radar cross-section of particular vessels is low, radar reflectors are placed on them. International standards specify under which conditions and at what sea state radar devices should be able to detect vessels.

Based on signals that are reflected from objects, a radar image is created and it represents the situation within radar coverage. As a result, one can track vessels, detect obstructions to navigation and see an image of the shoreline, etc. both under difficult weather conditions and when visibility is poor.

The radar subsystem that monitors vessel traffic in the Bay of Gdańsk, which is referred to as the VTS system for the Bay of Gdańsk, is an example of coastal radar stations functioning within VTS systems (Bole, Wall, Norris 2014). Figure 1 presents the distribution of coastal radar stations.

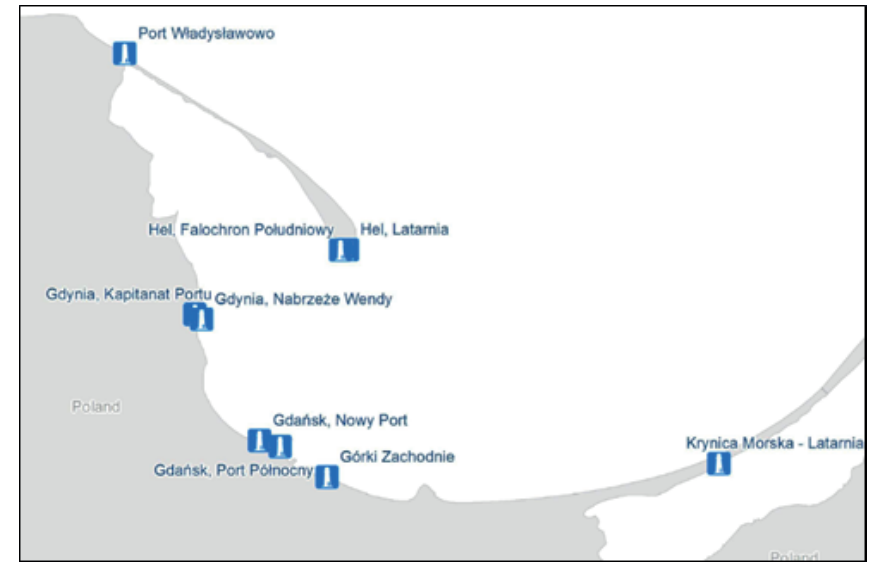

Fig. 1. Location of coastal radar stations functioning as part of the VTS system for the Bay of Gdanisk (source: Maritime Office in Gdynia)

Depending on the needs, information about a particular situation in a given sea area can be obtained from either one or several radars. The number and spatial arrangement of radars in VTS systems depends on the size and geospatial location of a given sea area. Radars are located around specific waters and they cover the whole area that is supervised by the VTS system. This is very important when return echoes from targets are 
weak or there has been a failure in one of the stations. In such cases one receives a large number of navigational data which can be used to improve the parameters (position) of a vessel by employing modern methods of adjustment calculus that are used in geodesy. If navigational information is received from many independent radar stations, this allows one to more accurately determine the positions of vessels in systems supervising vessel traffic (Monahan, 2008).

If a vessel's position is determined accurately, this also means that maritime safety is improved, which is particularly important in sea areas with a large volume of traffic where the risk of collision is relatively high. Since vessels move when sailing it is impossible to repeat measurements at the same position. Therefore, both classical estimation methods and modern

M-estimation methods that are used in geodesy can be utilised to improve the accuracy with which vessels' positions are determined (Czaplewski, 2004; Wiśniewski, 2009).

As for robust estimation, a key issue is to select the appropriate attenuation function. Several

M-estimation methods are used in adjustment calculus; these methods utilise different attenuation functions or weight functions. Among the most well-known methods of this kind are: Huber's method, Hampel's method and the Danish attenuation function (Borre, Jorgensen, Kubik, 1983; Hampel, Ronchetti, Rousseeuw, Stahel, 2005). They were applied in the process of determining the position fix of a vessel by using radar navigation methods.

\section{OBTAINING NAVIGATIONAL INFORMATION FROM RADAR OBSERVATIONS}

\section{USING THE DISTANCE}

Observations from coastal radar stations, where a vessel's position at sea is determined based on the measurement of distance, can be utilised to measure navigational parameters which are used to establish a vessel's position (Bole, Wall, Norris 2014). The measurement structure will depend on the number of radars that were used to make measurements. Figure 2 presents an example radar network. One can measure the distance to vessels from five coastal radar stations that are distributed around an area covered by the VTS system.



Fig. 2. Distance measurement structure (source: own work)
The distances that are measured from coastal stations with known geographic coordinates will help in determining a vessel's estimated position. This method, which is used based on observations made by coastal radars, is to allow one to correct the coordinates of a vessel's position that have been determined by an officer in charge of a navigational watch.

In this case, the adjustment problem entails determining the position fix of a vessel at sea (in a sea area covered by a VTS system) based on radar observations and the vessel's dead reckoning position that has been established by the officer in charge of a navigational watch on the ship.

The distances from coastal radar stations that have been measured allow one to define the system of observation equations for five coastal stations. The expected values can be determined by using the following formula (Wiśniewski, 2009):

$$
d_{i}^{o}=\left.\sqrt{\left(X_{S_{i}}-X_{Z_{j}}\right)^{2}+\left(Y_{S_{i}}-Y_{Z_{j}}\right)^{2}}\right|_{\substack{i=1, \ldots, 5 \\ j=1, \ldots, m}}
$$

where:

$\left(X_{S_{i}}, Y_{S_{i}}\right)$ - coordinates of radar stations' positions,

$\left(X_{Z_{j}}, Y_{Z_{j}}\right)$ - coordinates of the vessel's positions.

The system of linear adjustment equations takes the following form (Wiśniewski, 2009):

$$
v_{i j}=\frac{\partial d_{i j}}{\partial X_{Z_{j}}} \hat{d}_{X_{Z_{j}}}+\frac{\partial d_{i j}}{\partial Y_{Z_{j}}} \hat{d}_{Y_{Z_{j}}}+d_{i j}^{0}-\left.d_{i j}^{o b s}\right|_{\substack{i=1, \ldots, 5 \\ j=1, \ldots, m}}
$$

where:

$\mathrm{v}_{\mathrm{ji}}$ - adjustment of the bearing measured from the $\mathrm{i}$-th radar station $(i=1, \ldots, 5)$ to the $j$-th position of the vessel,

$d_{i j}^{0}$ - the expected value of the distance from a radar to the vessel for $Z_{j}^{0}\left(X_{Z_{j}}^{0}, Y_{Z_{j}}^{0}\right)$,

$d_{i j}^{o b s}$ - the observed value for $Z_{j}^{0}\left(X_{Z_{j}}^{0}, Y_{Z_{j}}^{0}\right)$,

$Z_{j}-j$-th position of the vessel $(j=1, \ldots, m)$.

\section{USING THE BEARINGS}

In order to determine a vessel's position at sea one can also use other observations, for example, bearings from coastal radar stations (Bole, Wall, Norris 2014). Figure 3 presents an example measurement structure for the case that is discussed here. For each of the measurements made by several coastal radar stations we obtain bearings having true value $\operatorname{NRij}(i=1, \ldots, 5, j=1, \ldots, m)$ for the $i$-th observation made relative to the vessel's $j$-th position. The results of observations (for example, NRijobs) having true vales are fraught with measurement errors, and it is often assumed that a measurement error is a random variable which has certain assumed descriptive parameters. Since there are errors, especially random ones, it may be difficult to determine the value of a measurement. The value of a measurement is actually a theoretical argument and it can only be estimated by using certain legitimate methods. The methods of estimating the descriptive parameters of random variables that have been developed in mathematical statistics provide a theoretical basis for the methods of adjusting the results of measurements (Wiśniewski, 2004; Jianjun, 1996). 


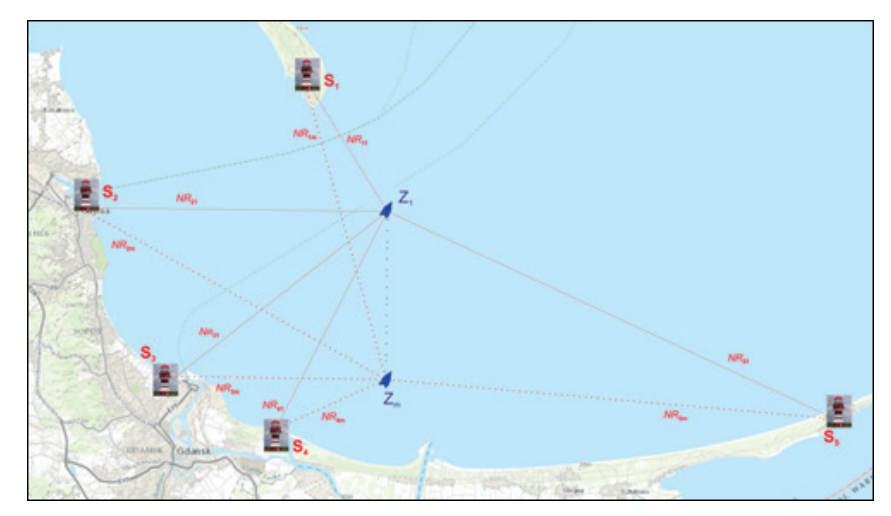

Fig. 3. Bearing measurement structure (source: own work)

Let us assume that we can use bearings that have been measured from coastal radar stations to a vessel at position $Z_{j}$. Then, the system of observation equations is as follows (Wiśniewski, 2009):

$$
N R_{i j}^{Z}=\left.\operatorname{arctg} \frac{Y_{S_{i}}-Y_{Z_{j}}}{X_{S_{i}}-X_{Z_{j}}}\right|_{\substack{i=1, \ldots, 5 \\ j=1, \ldots, m}}
$$

where:

$\left(X_{S_{i}}, Y_{S_{i}}\right)$ - coordinates of radar stations' positions,

$\left(X_{Z_{i}}, Y_{Z_{i}}\right)$ - coordinates of the vessel's positions.

For the measurement structure which is used for the purpose of this discussion and which is presented in Figure 3, the functional model of the adjustment problem takes the following form:

$$
v_{i j}=\frac{\partial N R_{i j}}{\partial X_{Z_{j}}} \hat{d}_{X_{Z_{j}}}+\frac{\partial N R_{i j}}{\partial Y_{Z_{j}}} \hat{d}_{Y_{Z_{j}}}+N R_{i j}^{0}-\left.N R_{i j}^{o b s}\right|_{\substack{i=1, \ldots, 5 \\ j=1, \ldots, m}} \text { (4) }
$$

where:

$N R_{i j}^{0}$ - the expected value of a radar bearing for $Z_{j}^{0}\left(X_{Z_{j}}^{0}, Y_{Z_{j}}^{0}\right)$ which was taken from the $i$-th radar station $(i=1, \ldots, 5)$ to the j-th position of the vessel,

$\mathrm{v}_{\mathrm{ij}}$ - adjustment to the observation that has been made.

In the adjustment process it is assumed that the position fix, which has been provided by an officer in charge of a navigational watch to a vessel traffic controller using the VTS system, is the expected position with the coordinates:

$$
\mathbf{Z}_{\mathbf{j}}^{\mathbf{0}}=\left[\begin{array}{l}
X_{Z_{j}}^{0} \\
Y_{Z_{j}}^{0}
\end{array}\right]
$$

Given that:

$$
\mathbf{V}=\left[\begin{array}{c}
v_{11} \\
\vdots \\
v_{i j}
\end{array}\right]_{\substack{i=1, \ldots, 5 \\
j=1, \ldots, m}} \text { - vector of adjustments, }
$$

$$
\hat{\mathbf{d}}_{X}=\left[\begin{array}{l}
\hat{d}_{X_{Z_{j}}} \\
\hat{d}_{Y_{Z_{j}}}
\end{array}\right] \text {-the unknown vector of increments to the }
$$

The matrix of coefficients of the unknowns and the matrix of constants assume different values, depending on the observations that are used. When it is distances that are used then we will obtain:

$$
\begin{aligned}
\mathbf{A} & =\left[\begin{array}{ll}
\frac{\partial d_{11}}{\partial X_{Z_{j}}} & \frac{\partial d_{11}}{\partial Y_{Z_{j}}} \\
\vdots & \vdots \\
\frac{\partial d_{i j}}{\partial d_{i j}} & \frac{-}{}
\end{array}\right] \\
\mathbf{L} & =\left[\begin{array}{l}
d_{11}^{0}-d_{11}^{o b s} \\
\vdots \\
d_{i j}^{0}-d_{i j}^{o b s}
\end{array}\right]_{\substack{i=1, \ldots, 5 \\
j=1, \ldots, m}} \text { - matrix of coefficients }
\end{aligned}
$$

However, if it is bearings that are used then:

$$
\begin{aligned}
& {\left[\begin{array}{ll}
\frac{\partial N R_{11}}{\partial X_{Z_{j}}} & \frac{\partial N R_{11}}{\partial Y_{Z_{j}}}
\end{array}\right] \quad \text { - matrix of coefficients }} \\
& \mathbf{A}=\left[\begin{array}{ll}
\frac{\partial X_{Z_{j}}}{\vdots} & \partial Y_{Z_{j}} \\
\frac{\partial N R_{i j}}{\partial X_{Z_{j}}} & \frac{\partial N R_{i j}}{\partial Y_{Z_{j}}}
\end{array}\right]_{\substack{i=1, \ldots, 5 \\
j=1, \ldots, m}} \\
& \text { of the unknowns, } \\
& \mathbf{L}=\left[\begin{array}{l}
N R_{11}^{0}-N R_{11}^{o b s} \\
\vdots \\
N R_{i j}^{0}-N R_{i j}^{o b s}
\end{array}\right]_{\substack{i=1, \ldots, 5 \\
j=1, \ldots, m}} \quad \text { - matrix of constants. }
\end{aligned}
$$

If we adopt the matrix structures that are presented above, i.e. A, $\hat{\mathbf{d}}_{X}$, L, the matrix system of adjustment equations takes the following form:

$$
\mathbf{V}=\mathbf{A} \cdot \hat{\mathbf{d}}_{X}+\mathbf{L}
$$

If the indefinite method is used, the solution to the system of equations will be as follows:

$$
\hat{\mathbf{d}}_{X}=-\left(\mathbf{A}^{T} \mathbf{P A}\right)^{-1} \mathbf{A}^{T} \mathbf{P} \mathbf{L}
$$

where:

$$
\mathbf{P}=\left[\begin{array}{ccc}
\frac{1}{m_{11}^{2}} & & \\
& \ddots & \\
& & \frac{1}{m_{i j}^{2}}
\end{array}\right]_{\substack{i=1, \ldots, 5 \\
j=1, \ldots, m}}
$$

- is a weight matrix of the observations made. 
Where:

$\mathrm{m}_{\mathrm{ij}}$ - average error of the $\mathrm{i}$-th observation made relative to the vessel's $j$-th position.

Therefore, the estimators of the adjusted coordinates of a vessel at sea are as follows:

$$
\hat{\mathbf{Z}}_{\mathbf{j}}=\mathbf{Z}_{\mathbf{j}}^{\mathbf{0}}+\hat{\mathbf{d}}_{\mathrm{XY}}=\left[\begin{array}{l}
X_{Z_{j}}^{0} \\
Y_{Z_{j}}^{0}
\end{array}\right]+\left[\begin{array}{l}
\hat{d}_{X_{Z_{j}}} \\
\hat{d}_{Y_{Z_{j}}}
\end{array}\right]=\left[\begin{array}{l}
\hat{X}_{Z_{j}} \\
\hat{Y}_{Z_{j}}
\end{array}\right]
$$

Where:

$$
\left[\begin{array}{l}
\hat{X}_{Z_{j}} \\
\hat{Y}_{Z_{j}}
\end{array}\right] \text { - estimated coordinates of a vessel at sea in the }
$$

In order to determine the quality of the observations that have been made, a statistical model of the adjustment problem should be defined:

$$
\hat{\mathbf{C}}_{\hat{\mathbf{x}}}=m_{0}^{2}\left(\mathbf{A}^{T} \mathbf{P A}\right)^{-1}=\left[\begin{array}{cc}
m_{\hat{X}_{Z_{j}}}^{2} & \hat{\operatorname{cov}}\left(\hat{X}_{j}, \hat{Y}_{j}\right) \\
\hat{\operatorname{cov}}\left(\hat{X}_{j}, \hat{Y}_{j}\right) & m_{\hat{Y}_{Z_{j}}}^{2}
\end{array}\right] \text { (9) }
$$

where:

$$
m_{0}^{2}=\frac{V^{T} P V}{n-r}-\text { estimator of the coefficient of variance, }
$$

$\mathrm{n}$ - number of observations made,

$\mathrm{r}$ - number of the unknowns.

Then the average error of the position fix will be determined based on the formula:

$$
M_{p o}=\sqrt{m_{\hat{X}_{Z_{j}}}^{2}+m_{\hat{Y}_{Z_{j}}}^{2}}
$$

If we assume that the goal function for the present research problem is compatible with the goal function in the method of least squares, the adjustment problem can be finally written as follows:

$$
\left\{\begin{array}{l}
\mathbf{V}=\mathbf{A} \cdot \hat{\mathbf{d}}_{X}+\mathbf{L} \\
\hat{\mathbf{C}}_{\hat{\mathbf{x}}}=m_{0}^{2}\left(\mathbf{A}^{T} \mathbf{P A}\right)^{-1} \\
\psi\left(\mathbf{V}^{T} \mathbf{P V}\right)=\min
\end{array}\right.
$$

\section{SELECTED ATTENUATION FUNCTIONS}

In marine navigation, the measurement structure can reflect the work of real devices that measure directions; then an angle is calculated between the direction vectors. Among such devices are the above-mentioned coastal radar stations, which are used to collect navigational data (for example, a bearing) that are needed to determine the positions of vessels in sea areas. Figure 3 presents an example structure of this kind.

The results of measurements can sometimes be fraught with serious errors. Such errors may occur, for example, because measuring instruments have been poorly fitted or calibrated or the indications have been read incorrectly, or due to temporary changes in the parameters of the measurement environment, etc. Errors of this kind are referred to as gross errors. In order to eliminate them, one can use robust estimation methods with properly selected attenuation functions.

Many M-estimation methods are used in adjustment calculus; these methods utilise different attenuation functions or different weight functions. Among the most well-known methods of this kind are: the Danish, Huber's, and Hampel's methods. For the purpose of this study, the three functions were analysed with regard to their influence on the accuracy with which positions are determined for measurements that are fraught with gross errors; these errors are described generally in the text above based on a paper written by Borre, Jorgensen, Kubik (1983), Teunissen P.J.G. (2004) and Hampel, Ronchetti, Rousseeuw, Stahel, (2005).

The characteristics of the Danish attenuation function are that it decreases exponentially outside acceptable range $\Delta \bar{v} \in\langle-k ; k\rangle$ and takes the following form:

$$
t(\bar{v})=\left\{\begin{array}{lll}
1 & \text { for } & \bar{v} \in\langle-k ; k\rangle \\
\exp \left[-l(|\bar{v}|-k)^{g}\right] & \text { for } & |\bar{v}|>k
\end{array}\right.
$$

Therefore, equivalent weight values will be determined according to this formula:

$$
\hat{p}=t(\bar{v}) p=\left\{\begin{array}{lll}
p & \text { for } & \bar{v} \in\langle-k ; k\rangle \\
\exp \left[-l(|\bar{v}|-k)^{g}\right] p & \text { for } & |\bar{v}|>k
\end{array}\right.
$$

The form of the Danish attenuation function is presented in Figure 4.

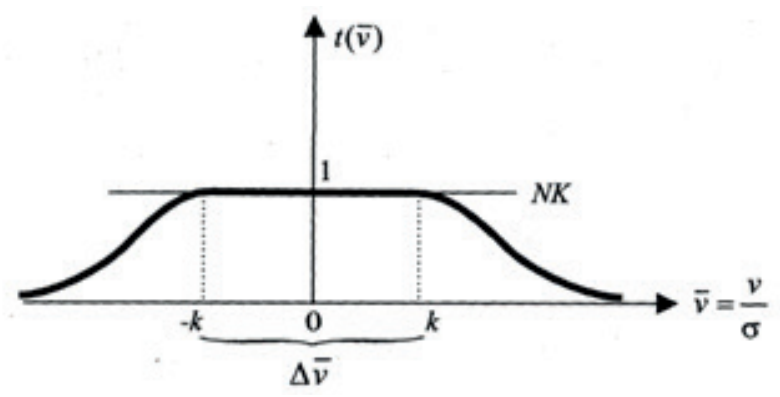

Fig. 4. Danish attenuation function (source: Wiśniewski, 2009)

Hampel's attenuation function has two additional intermediate ranges (to the left and to the right of acceptable range $\Delta \bar{v} \in\langle-k ; k\rangle)$ in which attenuation function $t(\bar{v})$ decreases linearly (Hampel, Ronchetti, Rousseeuw, Stahel, 2005). This function and the resulting weight function take the following forms:

$$
t(\bar{v})=\left\{\begin{array}{lll}
1 & \text { for } & \bar{v} \in\langle-k ; k\rangle \\
\frac{|\bar{v}|-k_{b}}{k-k_{b}} & \text { for } & |\bar{v}| \in\left(k ; k_{b}\right\rangle \\
0 & \text { for } & |\bar{v}|>k_{b}
\end{array}\right.
$$




$$
\hat{p}=t(\bar{v}) p=\left\{\begin{array}{lll}
p & \text { for } & \bar{v} \in\langle-k ; k\rangle \\
\left(\frac{|\bar{v}|-k_{b}}{k-k_{b}}\right) p & \text { for } & |\bar{v}| \in\left(k ; k_{b}\right\rangle \\
0 & \text { for } & |\bar{v}|>k_{b}
\end{array}\right.
$$

where $k_{b}$ is a number that determines the limits of the additional ranges. It is usually assumed that $\mathrm{k} \_\mathrm{b}=4, \ldots, 6$. The form of Hampel's attenuation function is presented in Figure 5.

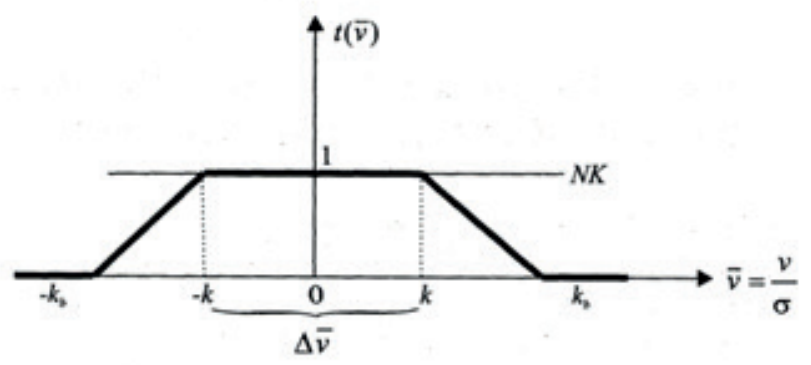

Fig. 5. Hampel's attenuation function (source: Wiśniewski, 2009)

Huber's attenuation function was the last of the analysed functions; this function assigns zero weight values to all observations with adjustments that do not belong to the acceptable range and it takes this form:

$$
t(\bar{v})=\left\{\begin{array}{lll}
1 & \text { for } & \bar{v} \in\langle-k ; k\rangle \\
0 & \text { for } & \bar{v} \notin(-k ; k)
\end{array}\right.
$$

Thus, the weight function is as follows:

$$
\hat{p}=t(\bar{v}) p=\left\{\begin{array}{llc}
p & \text { for } & \bar{v} \in\langle-k ; k\rangle \\
0 & \text { for } & \bar{v} \notin(-k ; k)
\end{array}\right.
$$

The form of Huber's attenuation function is presented in Figure 6.



Fig. 6. Huber's attenuation function (source: Wiśniewski, 2009)

Finally, the Danish attenuation function was selected because its form was most suited to dynamic models that perform typical navigation tasks.

If one uses a method that is robust to gross errors then the adjustment problem can be expressed in the following form:
$\mathbf{V}=\mathbf{A} \hat{\mathbf{d}}_{X}+\mathbf{L}$

$\mathbf{C}_{\mathbf{X}}=\sigma_{0}^{2} \mathbf{Q}_{\mathbf{X}}=\sigma_{0}^{2} \mathbf{P}^{-1}$

$\widehat{\mathbf{C}}_{\mathbf{X}}=\sigma_{0}^{2} \widehat{\mathbf{Q}}_{\mathbf{X}}=\sigma_{0}^{2} \widehat{\mathbf{P}}^{-1}$

$\widehat{\mathbf{P}}=\mathbf{T}(\overline{\mathbf{V}}) \mathbf{P}$

$\Psi\left(\hat{\mathbf{d}}_{X}\right)=\mathbf{V}^{T} \hat{\mathbf{P}} \mathbf{V}=\mathbf{V}^{T} \mathbf{T}(\overline{\mathbf{V}}) \mathbf{P V}=\min$

$\mathbf{V}^{T} \mathbf{T}(\overline{\mathbf{V}}) \mathbf{P V}=\hat{\mathbf{V}}^{T} \mathbf{T}(\hat{\overline{\mathbf{V}}}) \mathbf{P} \hat{\mathbf{V}}$

Where:

$\widehat{\mathbf{P}}=\mathbf{T}(\overline{\mathbf{V}}) \mathbf{P}-$ equivalent weight matrix,

$\hat{\mathbf{C}}_{\mathbf{X}}$ - equivalent covariance matrix,

$\hat{\mathbf{Q}}_{\mathbf{x}}$ - equivalent cofactor matrix,

$\mathbf{T}(\overline{\mathbf{V}})$ - diagonal attenuation matrix.

For such assumptions, the solution to the adjustment problem is iterative, i.e. the algorithm that was proposed in papers written by Wiśniewski $(2004,2009)$ can be used to solve this problem - at the first stage of calculations adjustment is made by using the classical method of least squares. An M-estimation method with the Danish attenuation function is presented in this paper.

\section{M-ESTIMATION WITH THE DANISH ATTENUATION FUNCTION}

As for the measurements of navigational parameters, the obtained results can be different than expected. If such errors, which are called gross errors, occur it is recommended that the measurement and results be corrected. It is, however, impossible to repeat a measurement for a moving vessel. At each time step, a vessel covers a certain distance and it "does not come back" to the point at which the last measurement was made. Serious errors can lead to using nautical charts for navigation incorrectly, and consequently pose a threat to maritime safety. It is possible to limit the influence of such errors on how a course line is drawn on a nautical chart by using modern M-estimation methods.

In a classical adjustment which is made by using the method of least squares, the results of measurements are random variables with the same standard deviation and they are treated equally; therefore, gross errors may significantly affect the measurement results. This method is not robust to serious errors. Thus, one can use robust estimation methods with properly selected attenuation functions in order to eliminate such errors. Later in the study the Danish attenuation function is used in the M-estimation procedure.

For this method, one can use the measurement structure which is shown in Figure 3 and which will be defined in the coordinate system (X, Y), where a bearing represents an observation. Radar observations can be made from coastal stations with regard to a vessel at position for a given measurement network. Bearings were measured at various time intervals.

If we assume that a given observation that has been made is fraught with gross error, for example, because the radar echo of a vessel was identified incorrectly, then a so-called 
equivalent weight $\widehat{\mathbf{P}}$ will be assigned to this observation; this weight will result from attenuating the original weight $\mathbf{P}$ (resulting from the assumed average measurement error). The attenuation process will occur in accordance with the following formula (Wiśniewski, 2009):

$$
\widehat{\mathbf{P}}=\mathbf{T}(\overline{\mathbf{V}}) \mathbf{P}
$$

where: $\mathbf{T}(\overline{\mathbf{V}})$ is an attenuation function having the following basic properties:

for $v_{i} \in \Delta v_{i}, v_{j} \in \Delta v_{j}: t\left(v_{i}\right)=t\left(v_{j}\right)$
for $\left(v_{i} \notin \Delta v_{i}\right) \vee\left(v_{j} \notin \Delta v_{j}\right),\left|v_{i}\right|<\left|v_{j}\right|: t\left(v_{i}\right)>t\left(v_{j}\right)$

Ranges $\Delta v_{i}=\left\langle-k \sigma_{v_{i}} ; k \sigma_{v_{i}}\right\rangle$ are acceptable ranges for adjustments $v_{\mathrm{i}}, \mathrm{i}=1, \ldots, \mathrm{n}$, and they are established with the assumed confidence level $\gamma$. If we assume that $v_{i}$ are random variables with a normal distribution, we can write the following:

$\gamma=P\left(-k \sigma_{v_{i}}<v_{i}<k \sigma_{v_{i}}\right)=P\left(-k<\bar{v}_{i}<k\right)=\int_{-k}^{+k} \frac{1}{\sqrt{2 \pi}} \exp \left[-\frac{\bar{v}_{i}^{2}}{2}\right] d \bar{v}_{i}$

where:

$\sigma_{v_{i}}=\sqrt{\left[\mathbf{C}_{V}\right]_{i i}}-$ standard deviation for the i-th adjustment,

$\bar{v}_{i}=\frac{v_{i}}{\sigma_{v_{i}}}$ - standardised adjustments with the same $v_{i}$ acceptable range $\Delta \bar{v}_{i}=<-k ; k>$. For example, for $\gamma=0.95$ coefficient $\mathrm{k}=2$.

Equivalent weight matrix $\widehat{\mathbf{P}}=\mathbf{T}(\overline{\mathbf{V}}) \mathbf{P}$ depends on the vector of standardised adjustments $\overline{\mathbf{V}}$. If a method that is robust to gross errors is used, the adjustment problem can be expressed as formula (18) (Wiśniewski, 2009; Yang, Song, Xu, 2002).

For such assumptions, the solution to the adjustment problem, which involves determining such $\hat{\mathbf{d}}_{X}$ that $\xi\left(\hat{\mathbf{d}}_{X}\right)=\mathbf{V}^{T} \hat{\mathbf{P}} \mathbf{V}=\min$, is iterative. In the first steps in the iterative process that is aimed to solve the adjustment problem, it is assumed that $1=0,01 \div 0,1, \mathrm{~g}=2$. If parameters are chosen incorrectly, this unnecessarily increases the number of steps in the iterative process which is aimed to solve a robust adjustment problem. A well-known algorithm was used to solve this problem; at the first stage bearings were adopted as measurement observations and adjustment was made by using the classical method of least squares (3) $\div(10)$. Further calculations allow us to establish which of the standardised adjustments can represent gross errors (they do not belong to $\Delta \bar{v}$ ) and determine a covariance matrix of the vector of adjustments for $\mathrm{m}_{0}=1$.

$$
\hat{\mathbf{C}}_{\hat{\mathbf{v}}\left(\mathrm{m}_{\mathrm{o}}=1\right)}=\mathbf{P}^{-1}-\mathbf{A}\left(\mathbf{A}^{T} \mathbf{P} \mathbf{A}\right)^{-1} \mathbf{A}^{T}
$$

Then we carry out classification in order to find out if for each:

$$
i=\bar{v}_{i} \quad \in \Delta \bar{v}
$$

If all $\bar{v}_{i} \in \Delta \bar{v}$ then we do not perform further calculations because the values of standardised adjustments that have been obtained fall within the acceptable range, and a new attenuation matrix will not reduce the values of weight matrices and then also the values of adjustments (within the precision range that has been assumed for the calculations).

Otherwise, if any adjustment $\bar{v}_{i} \notin \Delta v$, the next steps in the iterative process are performed (Wiśniewski, 2009):

We assume that $j=0$ and then $\mathbf{V}^{(j)}=\mathbf{V}, \mathbf{P}^{(j)}=\mathbf{P}, \mathbf{C}_{\mathbf{V}\left(m_{o}=1\right)}^{(j)}=\hat{\mathbf{C}}_{\hat{\mathbf{V}}\left(m_{o}=1\right)}^{(j)}$ and the control parameters in the Danish attenuation function $l=0.01, g=2$, and the following is calculated:

by remembering that:

$$
t(\bar{v})=\left\{\begin{array}{lll}
1 & \text { for } & \bar{v} \in\langle-k ; k\rangle \\
\exp \left[-l(|\bar{v}|-k)^{g}\right] & \text { for } & |\bar{v}|>k
\end{array}\right.
$$

we calculate the values of the attenuation function:

$$
\begin{aligned}
& \bar{v}_{i}^{(j)} \in \Delta \bar{v} \rightarrow t\left(\bar{v}_{i}^{(j)}\right)=1 \\
& \bar{v}_{i}^{(j)} \notin \Delta \bar{v} \rightarrow t\left(\bar{v}_{i}^{(j)}\right)=\exp \left[-l(|\bar{v}|-k)^{g}\right]
\end{aligned}
$$

and the attenuation matrix

$$
\mathbf{T}\left(\overline{\mathbf{V}}^{(j)}\right)=\left[\begin{array}{ccc}
\mathrm{t}\left(\bar{v}_{\mathrm{i}}^{(j)}\right) & & \\
& \ddots & \\
& & \mathrm{t}\left(\bar{v}_{\mathrm{n}}^{(j)}\right)
\end{array}\right]_{\mathrm{dla} a=1, \ldots, n}
$$

Then we perform iteration and increase $j$ by 1 , i.e. $j=j+1$, and we calculate weight matrices as well as matrix increases and adjustments:

$$
\left.\begin{array}{l}
\mathbf{P}^{(j)}=\mathbf{T}\left(\overline{\mathbf{V}}^{(j-1)}\right) \mathbf{P}^{(j-1)} \\
\mathbf{d}_{X}^{(j)}=-\left(\mathbf{A}^{T} \mathbf{P}^{(j)} \mathbf{A}\right)^{-1} \mathbf{A}^{T} \mathbf{P}^{(j)} \mathbf{L} \\
\mathbf{V}^{(j)}=\mathbf{A} \cdot \mathbf{d}_{X}^{(j)}+\mathbf{L}
\end{array}\right\}
$$

For the precision range that has been assumed for the calculations, the differences between elements of vectors of adjustments are checked:

$$
\mathbf{V}^{(j)} \text { i } \mathbf{V}^{(j-1)}
$$

If these differences are larger than assumed, we calculate a covariance matrix of the vector of adjustments for $m_{0}=1$

$$
\begin{aligned}
& \mathbf{C}_{\mathbf{V}}^{(j)}{ }_{\left(m_{o}=1\right)}=\left(\mathbf{P}^{(j)}\right)^{-1}-\mathbf{A}\left(\mathbf{A}^{T} \mathbf{P}^{(j)} \mathbf{A}\right)^{-1} \mathbf{A}^{T} \\
& m_{v_{i}}^{(j)}=\sqrt{\left[\mathbf{C}_{\mathbf{V}}^{(j)}{ }_{\left(m_{o}=1\right)}\right]_{i i}} \quad \bar{v}_{i}^{(j)}=\frac{v_{i}^{j}}{m_{v_{i}}^{(j)}}
\end{aligned}
$$


Then we calculate the value of the attenuation function and attenuation matrix. We increase $j$ by 1 , i.e. $j=j+1$, and proceed to the next iteration step. The iteration process ends with an adjustment whereby the obtained values of standardised adjustments fall within the acceptable range, and the resulting new attenuation matrix will not reduce the values of weight matrices and then also the values of adjustments (within the precision range that has been assumed for the calculations). The final weight matrix is an equivalent matrix, and the solution that was arrived at based on this matrix is the final solution. In the equivalent weight matrix, weights that correspond to observations which are fraught with gross errors are no longer original weights (with values resulting from average measurement errors), but weights with reduced values, which are sometimes equal to zero.

\section{NUMERICAL TEST}

For a measurement network consisting of five coastal radar stations observations were made relative to a vessel floating in the Bay of Gdańsk. Bearings were the navigational observations that were made. Since the measuring series was long, the paper presents measurements for ten positions of the vessel. Table 1 presents the values of these observations. For the purposes of this study, one of the measurements is "fraught" with gross error, which in practice may occur if a radar operator has taken an incorrect bearing or if the targeted object has been incorrectly identified. For all positions, a gross error has occurred in the bearing taken from the radar station in the port on the Hel Peninsula.

Table 2 presents the coordinates of the coastal radar stations from which bearings were taken.

Table 2. Coordinates of the coastal radar stations (Source: own work).

\begin{tabular}{|c|c|c|}
\hline $\begin{array}{c}\text { Item } \\
\text { no. }\end{array}$ & Coastal station & $\begin{array}{c}\text { Rectangular coordinates } \\
(\mathrm{X}, \mathrm{Y})\end{array}$ \\
\hline 1 & Hel Lighthouse & $\begin{array}{l}X=6052476.63 \\
Y=357945.55\end{array}$ \\
\hline 2 & $\begin{array}{c}\text { Gdynia_KP Harbour } \\
\text { Master }\end{array}$ & $\begin{array}{l}X=6045669.81 \\
Y=341309.47\end{array}$ \\
\hline 3 & $\begin{array}{c}\text { Gdynia_S Harbour } \\
\text { Master }\end{array}$ & $\begin{array}{l}X=6045119.44 \\
Y=342083.22\end{array}$ \\
\hline 4 & $\begin{array}{l}\text { Gdańsk North Port } \\
\text { Harbour Master }\end{array}$ & $\begin{array}{l}X=6031298.79 \\
Y=348189.74\end{array}$ \\
\hline 5 & $\begin{array}{l}\text { Radar Tower Górki } \\
\text { Zachodnie }\end{array}$ & $\begin{array}{l}X=6027017.31 \\
Y=355714.79\end{array}$ \\
\hline
\end{tabular}

Due to the large volume of research material, this paper only presents the results of the calculations for position $Z_{2}$. The results of all the adjustments that have been made are shown in Figure 7. In order to simplify the computational process, the authors decided that further calculations would

Table 1. Bearings, distances and coordinates for a vessel floating in the Bay of Gdańsk. (Source: own work).

\begin{tabular}{|c|c|c|c|c|c|c|}
\hline \multirow[b]{2}{*}{$\begin{array}{c}\text { Measurement } \\
\text { point }\end{array}$} & \multicolumn{5}{|c|}{ The coastal radar stations } & \multirow[b]{2}{*}{$Z_{\mathrm{j}}^{0}$} \\
\hline & $\begin{array}{c}\text { Hel } \\
\text { Lighthouse }\end{array}$ & $\begin{array}{c}\text { Gdynia_KP } \\
\text { Harbour } \\
\text { Master }\end{array}$ & $\begin{array}{c}\text { Gdynia_S } \\
\text { Harbour } \\
\text { Master }\end{array}$ & $\begin{array}{c}\text { Gdańsk } \\
\text { North Port } \\
\text { Harbour } \\
\text { Master }\end{array}$ & $\begin{array}{c}\text { Radar } \\
\text { Tower Górki } \\
\text { Zachodnie }\end{array}$ & \\
\hline $\mathrm{Z}_{1}$ & $213.9^{\circ}$ & $114.5^{\circ}$ & $113.1^{\circ}$ & $0.8^{\circ}$ & $334.4^{\circ}$ & $\begin{array}{l}X=6042478.33 \\
Y=348331.82\end{array}$ \\
\hline $\mathrm{Z}_{2}$ & $213.7^{\circ}$ & $114.6^{\circ}$ & $113.3^{\circ}$ & $0.7^{\circ}$ & $334.3^{\circ}$ & $\begin{array}{l}X=6042455.38 \\
Y=348340.88\end{array}$ \\
\hline $\mathrm{Z}_{3}$ & $213.7^{\circ}$ & $114.7^{\circ}$ & $113.3^{\circ}$ & $0.8^{\circ}$ & $334.3^{\circ}$ & $\begin{array}{l}X=6042436.72 \\
Y=348349.55\end{array}$ \\
\hline $\mathrm{Z}_{4}$ & $213.6^{\circ}$ & $114.8^{\circ}$ & $113.4^{\circ}$ & $0.8^{\circ}$ & $334.3^{\circ}$ & $\begin{array}{l}X=6042423.17 \\
Y=348355.15\end{array}$ \\
\hline $\mathrm{Z}_{5}$ & $213.5^{\circ}$ & $114.9^{\circ}$ & $113.6^{\circ}$ & $0.9^{\circ}$ & $334.3^{\circ}$ & $\begin{array}{l}X=6042389.72 \\
Y=348367.64\end{array}$ \\
\hline $\mathrm{Z}_{6}$ & $213.3^{\circ}$ & $115.2^{\circ}$ & $113.9^{\circ}$ & $1.0^{\circ}$ & $334.3^{\circ}$ & $\begin{array}{l}X=6042336.15 \\
Y=348387.79\end{array}$ \\
\hline $\mathrm{Z}_{7}$ & $213.2^{\circ}$ & $115.4^{\circ}$ & $114.2^{\circ}$ & $1.0^{\circ}$ & $334.3^{\circ}$ & $\begin{array}{l}X=6042313.62 \\
Y=348395.46\end{array}$ \\
\hline $\mathrm{Z}_{8}$ & $212.8^{\circ}$ & $116.0^{\circ}$ & $114.9^{\circ}$ & $1.3^{\circ}$ & $334.3^{\circ}$ & $\begin{array}{l}X=6042194.18 \\
Y=348437.39\end{array}$ \\
\hline $\mathrm{Z}_{9}$ & $212.3^{\circ}$ & $116.4^{\circ}$ & $115.3^{\circ}$ & $1.4^{\circ}$ & $334.2^{\circ}$ & $\begin{array}{l}X=6042121.38 \\
Y=348460.89\end{array}$ \\
\hline$Z_{10}$ & $211.8^{\circ}$ & $117.3^{\circ}$ & $116.3^{\circ}$ & $1.8^{\circ}$ & $334.2^{\circ}$ & $\begin{array}{l}X=6041954.26 \\
Y=348519.17\end{array}$ \\
\hline
\end{tabular}


be performed in a rectangular coordinate system and not in a geographic coordinate system.

The coordinates of a vessel's position that were determined by an officer in charge of a navigational watch are treated as the expected coordinates of a vessel's position at sea. For this observational arrangement, a system of adjustment equations is assumed, like in formula (4); after the above-mentioned data are substituted for the variables in this formula, the following elements of the matrix system of adjustment equations are obtained - formula (6).

When the observations that have been made are adjusted as part of the first step which is aimed at identifying gross errors by using the classical method, the following results are obtained:

The matrix of coefficients of the unknowns:

$$
\mathbf{A}=\left[\begin{array}{rr}
0.00286 & -0.00298 \\
-0.00674 & -0.00308 \\
-0.00775 & -0.00330 \\
-0.00007 & 0.00513 \\
0.00144 & 0.00302
\end{array}\right]
$$

The matrix of constants:

$$
\mathbf{L}=\left[\begin{array}{c}
223.78-213.75 \\
114.57-114.66 \\
113.06-113.29 \\
0.77-0.73 \\
334.47-334.33
\end{array}\right]=\left[\begin{array}{r}
10.03 \\
-0.09 \\
-0.23 \\
0.04 \\
0.014
\end{array}\right]
$$

If we assume that the average observation error is $m_{i j}=0.5^{\circ}$ for $i=1, \ldots, 5$ and that the observations that have been made are independent of one another, the weight matrix of the observations takes the following form:

$$
\mathbf{P}=\left[\begin{array}{lllll}
4 & 0 & 0 & 0 & 0 \\
0 & 4 & 0 & 0 & 0 \\
0 & 0 & 4 & 0 & 0 \\
0 & 0 & 0 & 4 & 0 \\
0 & & 0 & 0 & 4
\end{array}\right]
$$

The vector of increments to the expected coordinates is:

$$
\hat{\mathbf{d}}_{X}=-\left(\mathbf{A}^{T} \mathbf{P A}\right)^{-1} \mathbf{A}^{T} \mathbf{P} \mathbf{L}=\left[\begin{array}{l}
\hat{d}_{X_{Z_{2}}} \\
\hat{d}_{Y_{Z_{2}}}
\end{array}\right]=\left[\begin{array}{r}
-557.78 \\
796.05
\end{array}\right]_{[m]}
$$

Therefore, the vector of adjustments will take the value:

$$
\mathbf{V}=\left[\begin{array}{l}
v_{1} \\
v_{2} \\
v_{3} \\
v_{4} \\
v_{5}
\end{array}\right]=\left[\begin{array}{l}
6.07 \\
1.21 \\
1.47 \\
4.17 \\
1.74
\end{array}\right]
$$

Thus, the estimator of the position fix of a vessel at sea, which has been determined by using observations made from coastal radar stations, is:

$$
\begin{gathered}
\hat{\mathbf{Z}}_{1}=\left[\begin{array}{l}
\hat{X}_{Z_{1}} \\
\hat{Y}_{Z_{1}}
\end{array}\right]=\left[\begin{array}{l}
X_{Z_{1}}^{0} \\
Y_{Z_{1}}^{0}
\end{array}\right]+\left[\begin{array}{l}
\hat{d}_{X_{Z_{1}}} \\
\hat{d}_{Y_{Z_{1}}}
\end{array}\right]= \\
=\left[\begin{array}{r}
6042455.38 \\
348340.88
\end{array}\right]+\left[\begin{array}{r}
-557.78 \\
796.05
\end{array}\right]=\left[\begin{array}{r}
66041897.60 \\
349136.93
\end{array}\right]
\end{gathered}
$$

The estimators for the observations are, respectively:

$$
\left[\begin{array}{c}
\hat{N R_{1}} \\
\hat{N R_{2}} \\
\hat{N R_{3}} \\
\hat{N} R_{4} \\
\hat{N} R_{5}
\end{array}\right]=\left[\begin{array}{r}
219.8 \\
115.9 \\
114.8 \\
4.9 \\
336.1
\end{array}\right]_{\left[{ }^{\circ}\right]}
$$

The average error of the estimated coordinates of the vessel is, respectively:

$$
\begin{gathered}
m_{\hat{X}_{Z_{2}}}=\sqrt{1988}=478.1[\mathrm{~m}] \\
m_{\hat{Y}_{Z_{2}}}=\sqrt{4554}=639.3[\mathrm{~m}]
\end{gathered}
$$

Finally, the average error of the position is:

$$
M_{p o}=\sqrt{m_{\hat{\mathrm{X}}_{\mathrm{z}_{2}}}^{2}+m_{\hat{\mathrm{Y}}_{\mathrm{Z}_{2}}}^{2}}=798.3[\mathrm{~m}]
$$

Based on the obtained results, we must determine which of the standardised adjustments can represent gross errors. Let us assume, for the purpose of the calculations, that $\gamma=0.95$ , and thus $k=2$. The acceptable range $\Delta \bar{v}$ is as follows: $\Delta \bar{v} \in\langle-k ; k\rangle=\langle-2 ; 2\rangle$. In order to establish which of the standardised estimators of adjustments can represent gross errors (they do not belong to $\Delta \bar{v}$ ), we determine a covariance matrix of the estimator for the vector of adjustments $\hat{\mathbf{V}}$ for $m_{0}=1$

Then we carry out the classification:

$$
\hat{\mathbf{C}}_{\hat{\mathbf{v}}\left(\mathrm{m}_{\mathrm{o}}=1\right)}=\left[\begin{array}{rrrrr}
0.15130 & 0.02854 & 0.03774 & 0.10470 & 0.04162 \\
0.02854 & 0.14979 & -0.11445 & 0.01575 & 0.02913 \\
0.03774 & -0.11445 & 0.11899 & 0.01178 & 0.03044 \\
0.10470 & 0.01575 & 0.01178 & 0.11590 & -0.06476 \\
0.04162 & 0.02913 & 0.03044 & -0.06476 & 0.21402
\end{array}\right]
$$




$$
\begin{aligned}
& \overline{\hat{v}}_{1}=\frac{\left(\hat{v}_{1}\right)_{(m)}}{(\sqrt{0.15130})_{(m)}}=15.6 \quad \notin \Delta \bar{v} \\
& \overline{\hat{v}}_{2}=\frac{\left(\hat{v}_{2}\right)_{(m)}}{(\sqrt{0.14979})_{(m)}}=3.1 \quad \notin \Delta \bar{v} \\
& \overline{\hat{v}}_{3}=\frac{\left(\hat{v}_{3}\right)_{(m)}}{(\sqrt{0.11899})_{(m)}}=4.2 \quad \notin \Delta \bar{v} \\
& \overline{\hat{v}}_{4}=\frac{\left(\hat{v}_{4}\right)_{(m)}}{(\sqrt{0.11590})_{(m)}}=12.2 \quad \notin \Delta \bar{v} \\
& \overline{\hat{v}}_{5}=\frac{\left(\hat{v}_{5}\right)_{(m)}}{(\sqrt{0.21402})_{(m)}}=3.8 \quad \notin \Delta \bar{v}
\end{aligned}
$$

The obtained results show that none of the standardised estimators of adjustments belongs to the acceptable range. If we employed methods that are used in geodesy, we would have to reject measurements that are fraught with such errors and then repeat the measurements. However, it would be difficult to cause a vessel which is going to a port to return and then make the measurements for the same positions again. In order to neutralise these errors, we can adjust the results of measurements again, thus making the results of observations robust to gross errors, by using the Danish attenuation function. If we assume that $\mathbf{V}^{(0)}=\hat{\mathbf{V}}, \mathbf{P}^{(0)}=\hat{\mathbf{P}}$ and $l=0.02, g=2$ (control parameters in the Danish attenuation function), we can calculate the following: the value of the attenuation function (where $\Delta \bar{v} \in\langle-k ; k\rangle=\langle-2 ; 2\rangle$ );

$$
\begin{aligned}
& \bar{v}_{1}^{(0)}=\overline{\hat{v}}_{1} \notin \Delta \bar{v} \rightarrow t\left(\bar{v}_{1}^{(0)}\right)=\exp \left[-l(15.6-k)^{g}\right]=0.025 \\
& \bar{v}_{2}^{(0)}=\overline{\hat{v}}_{2} \notin \Delta \bar{v} \rightarrow t\left(\bar{v}_{2}^{(0)}\right)=\exp \left[-l(3.1-k)^{g}\right]=0.974 \\
& \bar{v}_{3}^{(0)}=\overline{\hat{v}}_{3} \notin \Delta \bar{v} \rightarrow t\left(\bar{v}_{3}^{(0)}\right)=\exp \left[-l(4.2-k)^{g}\right]=0.904 \\
& \bar{v}_{4}^{(0)}=\overline{\hat{v}}_{4} \notin \Delta \bar{v} \rightarrow t\left(\bar{v}_{4}^{(0)}\right)=\exp \left[-l(12.2-k)^{g}\right]=0.123 \\
& \bar{v}_{5}^{(0)}=\overline{\hat{v}}_{5} \notin \Delta \bar{v} \rightarrow t\left(\bar{v}_{5}^{(0)}\right)=\exp \left[-l(3.8-k)^{g}\right]=0.939
\end{aligned}
$$

and the attenuation matrix:

$$
\mathbf{T}\left(\overline{\mathbf{V}}^{(0)}\right)=\left[\begin{array}{lllll}
0.025 & 0 & 0 & 0 & 0 \\
0 & 0.974 & 0 & 0 & 0 \\
0 & 0 & 0.904 & 0 & 0 \\
0 & 0 & 0 & 0.123 & 0 \\
0 & 0 & 0 & 0 & 0.939
\end{array}\right]
$$

Then we perform calculations by using the iteration method; as part of step no. 1 we calculate the following: the attenuation matrix;

$$
\widehat{\mathbf{P}}^{(1)}=\mathbf{T}\left(\overline{\mathbf{V}}^{(0)}\right) \mathbf{P}^{(0)}=\left[\begin{array}{lllllr}
0.09897 & 0 & 0 & 0 & 0 \\
0 & 3.89754 & 0 & 0 & 0 \\
0 & 0 & 3.61422 & 0 & 0 \\
0 & 0 & 0 & 0.49091 & 0 \\
0 & 0 & 0 & 0 & 3.75768
\end{array}\right]
$$

coordinates' increments:

$$
\hat{\mathbf{d}}_{X}^{(1)}=-\left(\mathbf{A}^{T} \widehat{\mathbf{P}}^{(1)} \mathbf{A}\right)^{-1} \mathbf{A}^{T} \widehat{\mathbf{P}}^{(1)} \mathbf{L}=\left[\begin{array}{r}
-72.92 \\
89.62
\end{array}\right]_{(\mathrm{m})}
$$

the vector of adjustments;

$$
\hat{\mathbf{V}}^{(1)}=\mathbf{A} \cdot \hat{\mathbf{d}}_{X}^{(1)}+\mathbf{L}=\left[\begin{array}{c}
9.56 \\
0.12 \\
0.04 \\
0.51 \\
0.31
\end{array}\right]_{[0]}
$$

diagonal elements of matrix $\mathrm{C}_{\mathrm{V}\left(m_{0}=1\right)}^{(1)}$

$$
\mathbf{C}_{\mathbf{V}\left(m_{0}=1\right)}^{(1)}=\left[\begin{array}{rrrrr}
9.566 & 0.057 & 0.095 & 0.619 & 0.271 \\
0.057 & 0.144 & -0.130 & -0.012 & 0.019 \\
0.095 & -0.130 & 0.124 & -0.049 & 6.165 \cdot 10^{-3} \\
0.619 & -0.012 & -0.049 & 1.296 & -0.336 \\
0271 & 0.019 & 6.165 \cdot 10^{-3} & -0.336 & 0.108
\end{array}\right]
$$

the value of the attenuation function;

$$
\begin{array}{ll}
v_{1}^{(1)}=3.09 & \notin \Delta v \rightarrow \mathrm{t}\left(v_{1}^{(1)}\right)=\exp \left[-l(3.09-k)^{g}\right]=0.997 \\
\bar{v}_{2}^{(1)}=0.33 & \in \Delta \bar{v} \rightarrow \mathrm{t}\left(\bar{v}_{2}^{(1)}\right)=1 \\
\bar{v}_{3}^{(1)}=0.11 & \in \Delta \bar{v} \rightarrow \mathrm{t}\left(\bar{v}_{3}^{(1)}\right)=1 \\
\bar{v}_{4}^{(1)}=0.44 & \in \Delta \bar{v} \rightarrow \mathrm{t}\left(\bar{v}_{4}^{(1)}\right)=1 \\
\bar{v}_{5}^{(1)}=0.94 & \in \Delta \bar{v} \rightarrow \mathrm{t}\left(\bar{v}_{5}^{(1)}\right)=1
\end{array}
$$

and the attenuation matrix

$$
\mathbf{T}\left(\overline{\mathbf{V}}^{(1)}\right)=\left[\begin{array}{lllll}
0.977 & 0 & 0 & 0 & 0 \\
0 & 1 & 0 & 0 & 0 \\
0 & 0 & 1 & 0 & 0 \\
0 & 0 & 0 & 1 & 0 \\
0 & 0 & 0 & 0 & 1
\end{array}\right]
$$


Since only one standardised adjustment falls within the acceptable range, the iterative process of solving the adjustment problem by using a method that is robust to gross errors can be accelerated by adopting increasingly higher values of parameter $l$. Finally, the following solution is obtained:

$$
\begin{gathered}
\hat{\mathbf{d}}_{X}^{(1)}=-\left(\mathbf{A}^{T} \widehat{\mathbf{P}}^{(1)} \mathbf{A}\right)^{-1} \mathbf{A}^{T} \widehat{\mathbf{P}}^{(1)} \mathbf{L}=\left[\begin{array}{r}
-36.07 \\
19.87
\end{array}\right]_{(\mathrm{m})} \\
\hat{\mathbf{V}}^{(1)}=\mathbf{A} \cdot \hat{\mathbf{d}}_{X}^{(1)}+\mathbf{L}=\left[\begin{array}{r}
9.87 \\
0.09 \\
-0.02 \\
0.15 \\
0.15
\end{array}\right]_{\left[{ }^{\circ}\right]} \\
m_{\hat{X}_{Z_{2}}}=108.68[\mathrm{~m}] \\
m_{\hat{Y}_{Z_{2}}}=195.80[\mathrm{~m}] \\
M_{p o}=\sqrt{m_{\hat{\mathrm{X}}_{Z_{2}}}^{2}+m_{\hat{\mathrm{Y}}_{Z_{2}}}^{2}}=223.94[\mathrm{~m}]
\end{gathered}
$$

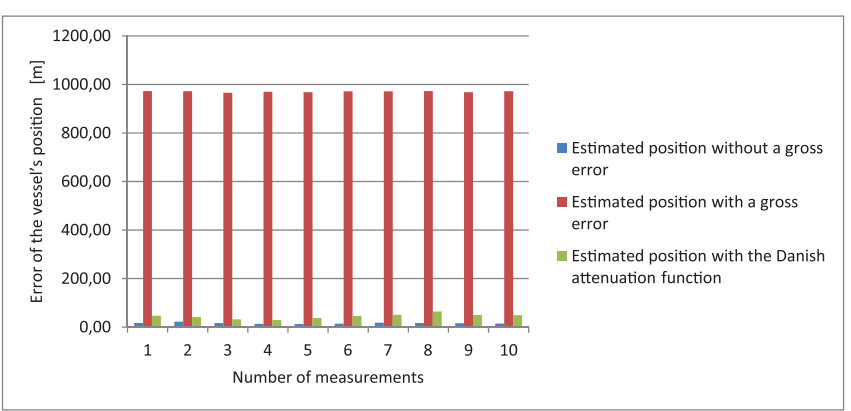

Figure 7. Error of the vessel's position which was determined during the study (source: own work).

\section{CONCLUSIONS}

Radar is one of the basic tools which are used in marine and land navigation and which makes it possible to visualise the surrounding area. This tool is used as one of the main sources of the information about vessel traffic at sea that is passed on to vessel traffic monitoring centres. If information about moving objects is obtained from several radar stations, this may be a step forward in assessing maritime safety since more complete and more accurate data will be available about vessel traffic in waters that are monitored by radar stations. Once it is possible to determine vessels' exact positions, maritime safety in coastal waters will improve because information that is necessary for safe navigation will be sent to the bridge.

When measuring the parameters that are used to determine a vessel's position, we can obtain results which will be fraught with serious errors if the measurement process is disturbed, and in extreme cases such errors can largely deviate from the expected results.
Consequently, if a vessel's position is erroneously determined, nautical charts may be incorrectly used for navigation and this can pose a threat to maritime safety.

The authors are not familiar with any other solutions which would involve simultaneously using information obtained from all coastal radar stations operating within the VTS system as well estimation methods that are employed in geodesy. At the same time, the use of the whole body of information that is provided by VTS systems as well as of modern M-estimation methods opens up new possibilities for the VTS system operators as regards traffic control and updating the positions of vessels in waters that are monitored by systems supervising vessel traffic.

In order to reduce the impact of gross errors on the process of establishing a vessel's position, one can use robust estimation methods with properly selected attenuation functions; this will determine the accuracy with which the position will be established. M-estimation methods can significantly correct such errors and limit their influence on how a course line is drawn on a nautical chart. This paper presents basic robust adjustment methods. The analysis has shown that a method utilising the Danish attenuation function is best suited to the case that is presented here. If the other, i.e. Hampel's and Hubert's, attenuation functions are used when serious errors occur, this leads to an unexpected shift in a vessel's position.

\section{REFERENCES}

1. Bole A.G., Wall A.W., Norris A., (2014). Radar and ARPA Manual, Third Edition: Radar, AIS and Target Tracking for Marine Radar Users 3rd Edition, Elsevier, ISBN 978-0-08097752-2, Waltham, MA.

2. Borre K.J, Jorgensen P.C., Kubik K., (1983). Robust Adjustment of the Danish Fundamental Triangulation Network. Zeszyty Naukowe Akademii Górniczo Hutniczej im. Stanisława Staszica, nr 79, s. 57 - 69, Kraków.

3. Czaplewski K. (2004). Positioning With Interactive Navigational Structures Implementation. Annual of Navigation no 7/2004, ISSN 1640-8632, Gdynia.

4. Hampel F.R, Ronchetti E.M, Rousseeuw P.J, Stahel W.A, (2005). Robust Statistics. The Approach Based on Influence Functions. John Wiley, ISBN 978-0-471-735779, New York, NJ

5. Jianjun Z. (1996). Robustness and the Robust Estimate. Journal of Geodesy, nr 70, s. 586 - 590, Heidelberg.

6. Monahan K. (2008) The Radar Book: Effective Navigation and Collision Avoidance, Fine Edge Productions, ISBN 978-1-932310-36-8, Anacortes, WA.

7. Teunissen P.J.G. (2004). Adjustment theory: an introduction (mathematical geodesy and positioning), Delft University Press, ISBN 978-90-407-1974-5, Delft. 
8. Wiśniewski Z. (2004). Metody opracowania wyników pomiarów w nawigacji i hydrografii. AMW, ISBN 8387280-65-8, Gdynia.

9. Wiśniewski Z. (2009). Rachunek wyrównawczy w geodezji (z przykładami). UWM, ISBN 83-7299-399-8, Olsztyn.

10. YangY., Song L., Xu T., (2002). Robust Estimator for Correlated Observations Based on Bifactor Equivalent Weights. Journal of Geodesy, nr 76, s. 353 - 358, Heidelberg.

\section{CONTACT WITH THE AUTHORS}

Sławomir Świerczyński

Polish Naval Academy

s.swierczynski@amw.gdynia.pl

Krzysztof Czaplewski

Gdynia Maritime University

krzysztof@czaplewski.pl

POLAND 